\title{
Pressure Induced Suppression to the Valence Change Transition in EuPdAs
}

\author{
Baoxuan Li, Jianzhong Liu, Yufeng Li, Xiyu Zhu, and Hai-Hu Wen \\ Center for Superconducting Physics and Materials, National Laboratory of Solid State Microstructures and Department of Physics, \\ National Center of Microstructures and Quantum Manipulation, Nanjing University, Nanjing 210093, China
}

Correspondence should be addressed to Hai-Hu Wen; hhwen@nju.edu.cn

Received 6 December 2014; Accepted 8 February 2015

Academic Editor: Viorel Sandu

Copyright (C) 2015 Baoxuan Li et al. This is an open access article distributed under the Creative Commons Attribution License, which permits unrestricted use, distribution, and reproduction in any medium, provided the original work is properly cited.

\begin{abstract}
By applying a hydrostatic pressure, we have successfully suppressed the valence change transition in EuPdAs. The studied compound EuPdAs crystallizes in a P63/mmc space group. Through resistivity and magnetic susceptibility measurements, we find that EuPdAs shows a phase transition at $180 \mathrm{~K}$ and another transition below $10 \mathrm{~K}$ at ambient pressure, as was reported before. The overall transport and magnetic behavior is to some extent similar to that of the parent phase of iron based superconductors. With application of a hydrostatic pressure, the transition at $180 \mathrm{~K}$ is sensitively suppressed with a pressure as low as $0.48 \mathrm{GPa}$. However, superconductivity has not been induced with pressure up to $1.90 \mathrm{GPa}$.
\end{abstract}

\section{Introduction}

The EuPdAs alloy adopts the hexagonal $\mathrm{CaIn}_{2}$-type structure. The transport and magnetic measurements of this material reveal a similarity to the parent phase of iron based superconductors $[1,2]$. Particularly, there is a clear transition at around $180 \mathrm{~K}$, which is of first order and accompanied with a large volume collapse caused by a strong contraction of the $c$-axis [3-5]. The transition at $180 \mathrm{~K}$ was reported to be induced by the fluctuation of Eu valence from 2.15 to 2.40 between $300 \mathrm{~K}$ and $4 \mathrm{~K}$ as revealed by experiments of Moessbauer measurement and $\mu \mathrm{SR}$ method $[3,6,7]$. However, whether this transition is related to some kind of magnetic transition is still unclear. And as it is known in the iron based superconductors, there is a close relationship between the high temperature phase transition and the emergence of superconductivity $[8,9]$. Inspired by this similarity, we intend to check whether the transition at $180 \mathrm{~K}$ can be suppressed under hydrostatic pressure, as many iron-based superconductors do, and, if the transition is really related to a certain magnetic transition, whether superconductivity can be induced with the suppression of the transition.

\section{Experimental Details}

The polycrystalline sample of EuPdAs was synthesized by solid state reaction method, using EuAs as the precursor made by reaction of Eu and As (purity 99.9\%, Alfa Aesar). High purity EuAs and Pd powder (99.99\% purity, Cuibolin) were weighed in stoichiometric ratio, mixed well, and pressed into a pellet in an argon filled glove box. The pellet was loaded into an alumina crucible, which was sealed in an evacuated quartz ampule and then kept inside a PID controlled furnace. The temperature of the furnace was raised to $1000^{\circ} \mathrm{C}$ with a heating rate of $1.2^{\circ} \mathrm{C} / \mathrm{min}$ and maintained for 24 hours. The sintered sample was cooled down naturally to room temperature with the furnace power turned off from $1000^{\circ} \mathrm{C}$.

$\mathrm{X}$-ray diffraction (XRD) measurements were performed on a Bruker D8 advanced diffractometer with the $\mathrm{Cu}-\mathrm{K}_{\alpha}$ radiation. Dc magnetization measurements were carried out with a SQUID-VSM-7T (Quantum Design). Measurements of resistivity under pressure were performed up to $2.3 \mathrm{GPa}$ on a physical property measurement system (PPMS-16T, Quantum Design) by using HPC-33 piston type pressure cell with the Quantum Design dc resistivity and ac transport options. For the resistive measurements, silver leads with a diameter of $50 \mu \mathrm{m}$ were glued to the EuPdAs sample in a standard four-probe method by using silver epoxy, and the sample was immersed in the pressure transmitting medium (Daphne 7373 ) in a Teflon cap with a diameter of $4 \mathrm{~mm}$. Hydrostatic pressure was generated by a $\mathrm{BeCu} / \mathrm{NiCrAl}$ clamped pistoncylinder cell. The pressure upon the sample was calibrated 


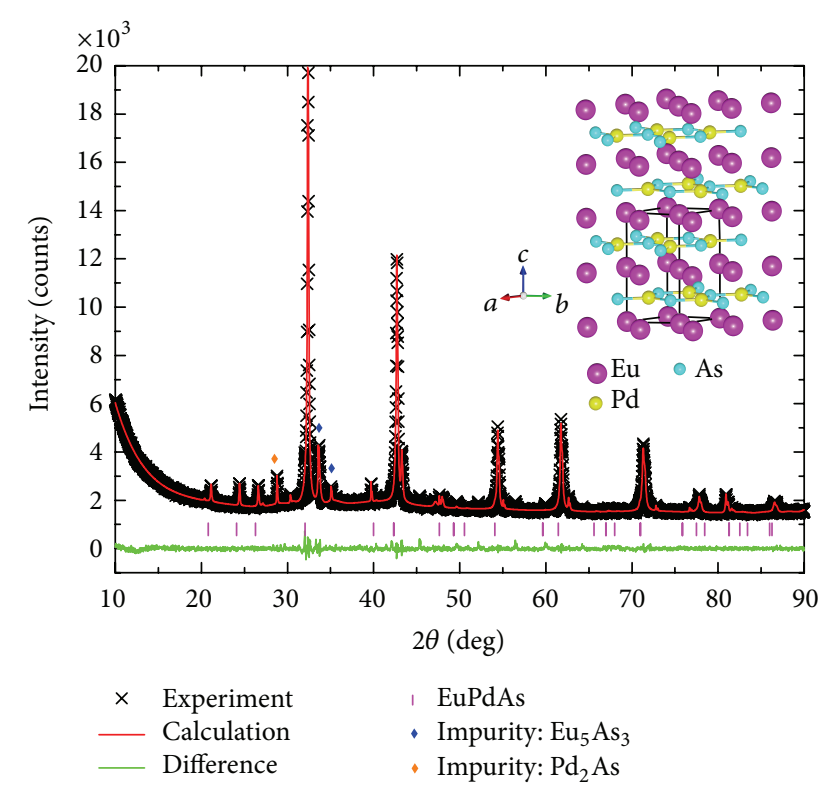

FIGURE 1: Powder X-ray diffraction patterns and the Rietveld refinement profile of the EuPdAs sample at room temperature. All main diffraction peaks can be indexed well by the $\mathrm{P} 63 / \mathrm{mmc}$ space group (hexagonal structure) with $a=4.262 \AA$ and $c=8.535 \AA$ with $\mathrm{Eu}_{5} \mathrm{As}_{3}$ and $\mathrm{Pd}_{2} \mathrm{As}$ as the impurity phases. The ratio between EuPdAs and the impurities is found to be $90: 10$.

with the shift in $T_{c}$ of a high purity $\mathrm{Sn}$ sample by measuring the temperature dependence of resistivity.

\section{Results and Discussion}

Figure 1 shows the powder XRD patterns of the EuPdAs sample at room temperature, along with the result of the Rietveld structural refinement using the TOPAS program. On the whole, it is clear that the main diffraction peaks can be indexed well by a hexagonal cell structure with the P63/mmc space group. In addition to a principal phase, some weak peaks arising from the impurity phases of $\mathrm{Eu}_{5} \mathrm{As}_{3}$ and $\mathrm{Pd}_{2} \mathrm{As}$ can also be seen. A detailed fitting to the structural data shows that the ratio between EuPdAs and the impurity phases is around $90: 10$ for this sample. The refined lattice parameters are extracted to be $a=4.262 \AA$ and $c=8.535 \AA$.

Figure 2(a) shows the temperature dependence of zerofield-cooled (ZFC) and field-cooled (FC) dc magnetic susceptibility for EuPdAs under 20 Oe. An anomaly can be clearly seen around $8 \mathrm{~K}$, where the ZFC curve shows some deviation from the FC curve; this may be an indication of long range magnetic ordering of Eu ions. We can also observe a kink at around $5.4 \mathrm{~K}$, which has not been reported before. We conjecture that the $5.4 \mathrm{~K}$ kink may be a secondary transition of the Eu ions ordering, but we cannot rule out the possibility that this anomaly arises from the impurity phases of $\mathrm{Eu}_{5} \mathrm{As}_{3}$ or $\mathrm{Pd}_{2}$ As. Figure 2(b) shows the temperature dependence of ZFC magnetic susceptibility under $1 T$. In addition to the susceptibility saturation below $10 \mathrm{~K}$, corresponding to the $8 \mathrm{~K}$ anomaly in Figure 2(a), there is another kink around $180 \mathrm{~K}$, which can be easily observed in the inset of Figure 2(b). And above $180 \mathrm{~K}$, the magnetic susceptibility of EuPdAs follows Curie-Weiss law behavior very well.

The temperature dependence of electrical resistivity for EuPdAs with temperature ranging from $2.3 \mathrm{~K}$ to $300 \mathrm{~K}$ is shown in Figure 3 . The $8 \mathrm{~K}$ and $180 \mathrm{~K}$ kinks also clearly appear in these resistivity curves, which is consistent with the magnetic measurements (while the $5.4 \mathrm{~K}$ anomaly is absent in the resistive curve). The overall transport behavior, especially the $180 \mathrm{~K}$ anomaly, reminds us of some parent phases of ironbased materials, like $\mathrm{BaFe}_{2} \mathrm{As}_{2}$, which exhibits antiferromagnetic order originated from $\mathrm{Fe}$ ions around $140 \mathrm{~K}$. When we apply pressure or do some chemical substitutions on $\mathrm{BaFe}_{2} \mathrm{As}_{2}$, the $140 \mathrm{~K}$ transition will be gradually suppressed to lower temperature, eventually, the antiferromagnetic transition will totally disappear and superconducting state will be induced $[2,10]$.

Inspired by the knowledge in the parent phase of iron based superconductors, we suspect that the $180 \mathrm{~K}$ anomaly for EuPdAs may be related to some kind of magnetic order of the Pd ions. If we can find some way to suppress this transition, we may expect some interesting properties, like superconductivity. To realize this purpose, we try to apply a hydrostatic pressure to EuPdAs.

The temperature dependence of resistivity for EuPdAs at various pressures with temperature ranging from $2.3 \mathrm{~K}$ to $250 \mathrm{~K}$ is illustrated in Figure 4. We find that the transition at $180 \mathrm{~K}$ is totally suppressed with a pressure of $0.48 \mathrm{GPa}$, and the resistivity value is depressed monotonously upon increasing pressure. However, no superconducting transition has been observed with pressure up to $1.90 \mathrm{GPa}$, while the $8 \mathrm{~K}$ anomaly is always preserved. Since the $180 \mathrm{~K}$ transition is so sensitive to pressure, we deem that it is merely a valence change transition and may not relate to the formation of any long range magnetic order. In addition, the $180 \mathrm{~K}$ anomaly can completely recover when pressure is removed (see Figure 3, the red curve); this reminds us of some pressure-induced iron-based superconductors, like $\mathrm{CaFe}_{2} \mathrm{As}_{2}$, in which the crystal structure will completely recover with pressure removed [11].

It is reported that the transition at $180 \mathrm{~K}$ is accompanied with a giant volume collapse of $1.9 \%$ caused by a strong contraction of the $c$-axis (2.3\%) between $180 \mathrm{~K}$ and $150 \mathrm{~K}$ [3$5]$; on the other hand, the lattice parameters will experience a strong but continuous decrease with increasing pressure [12]. Under the above considerations, it is reasonable to assume that the pressure we applied can result in the volume collapse at room temperature, which may induce the valence shift to 2.4 to occur at much higher temperature. Thus when applying pressure, the behaviour of the resistivity curve is well preserved below $180 \mathrm{~K}$, while the $180 \mathrm{~K}$ transition disappears and the curve above $180 \mathrm{~K}$ extends smoothly from that at lower temperature.

The resistivity of the system is generally about 100$300 \mu \Omega \mathrm{cm}$, which manifests that the sample can be categorized as a bad metal. Since Eu has usually an ionic state of $\mathrm{Eu}^{2+}$ and/or $\mathrm{Eu}^{3+}$, while the As can stay at several anionic states such as $\mathrm{As}^{3-}$ and $\mathrm{As}^{1-}$, in this case the ionic state of Pd can be 


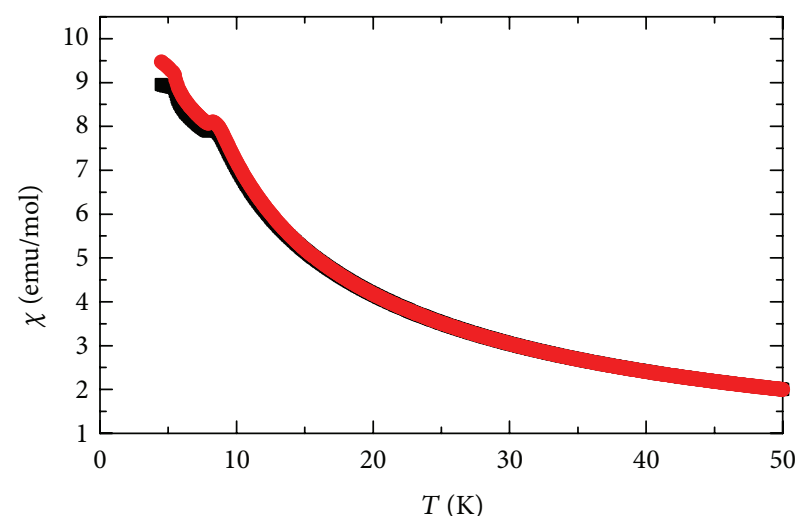

-- ZFC $20 \mathrm{Oe}$

- FC $20 \mathrm{Oe}$

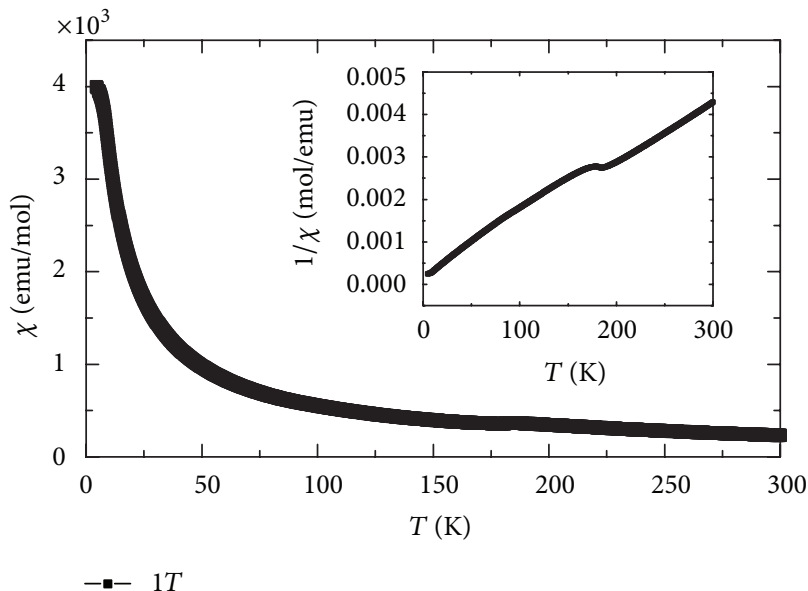

(b)

Figure 2: (a) Temperature dependence of dc magnetic susceptibility for EuPdAs as measured at an applied magnetic field of 20 Oe in the temperature range of $4.5 \mathrm{~K}$ to $50 \mathrm{~K}$. Both magnetic susceptibilities measured in zero-field-cooled (ZFC) and field-cooled (FC) modes are shown. (b) Temperature dependence of dc magnetic susceptibility for EuPdAs under $1 T$ in the temperature range of $4.5 \mathrm{~K}$ to $300 \mathrm{~K}$. The inset shows $1 / \chi$ versus $T$ to clarify the $180 \mathrm{~K}$ anomaly.

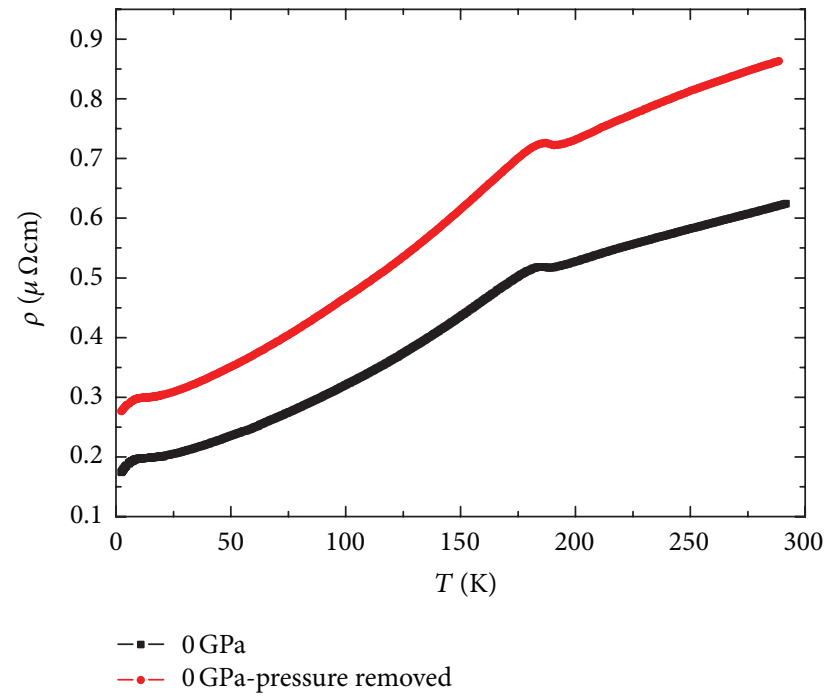

FIGURE 3: Temperature dependence of electrical resistivity for EuPdAs in the temperature range $2.3 \mathrm{~K}$ to $300 \mathrm{~K}$ (the black curve). The red curve is the result measured after pressure is removed (sample still immersed in the pressure transmitting medium).

widely tuned. This provides a good way to tune the electronic states of the materials. This may provide an explanation to the transition at around $180 \mathrm{~K}$, which is most probably induced by a valence change effect. From our recent study, one can see that the magnetic moment detected in the material is quite weak, either from the Pd or Eu ions. Nor has the long range magnetic order formation been found on the Pd ions. It would be interesting to use chemical doping to tune the electronic state $4 \mathrm{~d}$ orbital in order to achieve a good balance between the itinerancy and local magnetism.

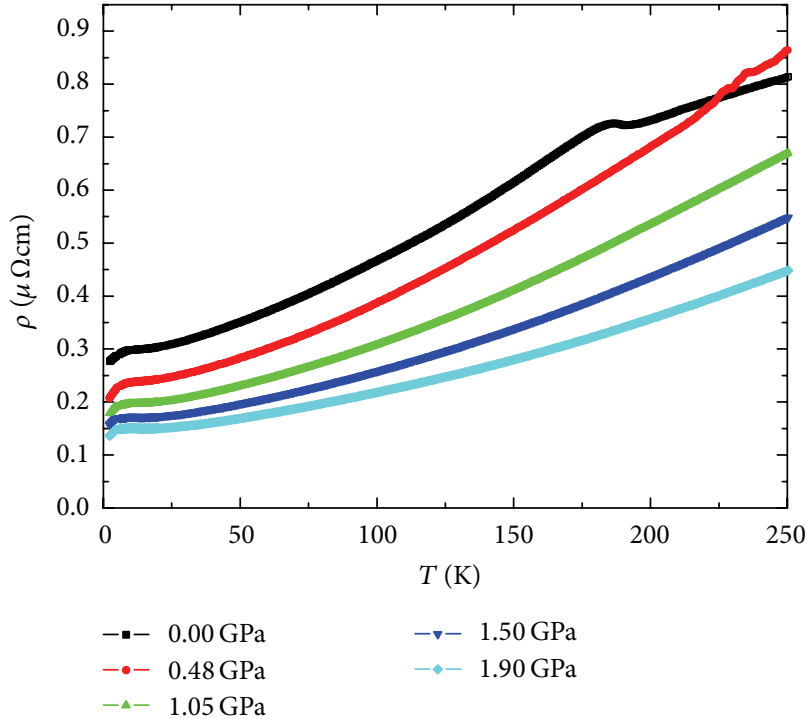

FIgURE 4: Temperature dependence of electrical resistivity for EuPdAs at various pressures in the temperature range $2.3 \mathrm{~K}$ to $250 \mathrm{~K}$.

\section{Conclusion}

In summary, we have synthesized polycrystalline samples of EuPdAs by solid state reaction method. Through resistivity and magnetic susceptibility measurements, we find that EuPdAs shows a phase transition at $180 \mathrm{~K}$, as well as a second phase transition below $10 \mathrm{~K}$ at ambient pressure. With application of hydrostatic pressure, the transition at $180 \mathrm{~K}$ is totally suppressed under a pressure of $0.48 \mathrm{GPa}$, and the resistivity curve is depressed monotonously upon increasing pressure. However, superconductivity has not been induced at low temperature with pressure up to $1.90 \mathrm{GPa}$. In order to achieve 
some novel electronic properties, such as superconductivity, we suggest that tuning the occupation of the $4 \mathrm{~d}$ orbitals of Pd with electrons would be essential.

\section{Conflict of Interests}

The authors declare that there is no conflict of interests regarding the publication of this paper.

\section{References}

[1] L. J. Li, Y. K. Luo, Q. B. Wang et al., "Superconductivity induced by $\mathrm{Ni}$ doping in $\mathrm{BaFe}_{2} \mathrm{As}_{2}$ single crystals," New Journal of Physics, vol. 11, Article ID 025008, 2009.

[2] P. C. Canfield and S. L. Bud'ko, "FeAs-based superconductivity: a case study of the effects of transition metal doping on $\mathrm{BaFe}_{2} \mathrm{As}_{2}$," Annual Review of Condensed Matter Physics, vol. 1, pp. 27-50, 2010.

[3] G. Michels, S. Junk, N. Lossau et al., "Mixed valency and first order phase transition in EuPdAs," Zeitschrift für Physik B Condensed Matter, vol. 86, pp. 53-58, 1992.

[4] D. Johrendt and A. Mewis, "Darstellung und kristallstrukturen der verbindungen SEPdAs (SELa-Lu)," Journal of Alloys and Compounds, vol. 183, pp. 210-223, 1992.

[5] G. Michels, C. Huhnt, W. Scharbrodt et al., "Temperature and pressure driven valence change in ternary Eu-pnictides," Physica B: Physics of Condensed Matter, vol. 206-207, pp. 408411, 1995.

[6] H.-H. Klauß, M. Hillberg, W. Wagener et al., "Valence fluctuations in ternary europium compounds," Hyperfine Interactions, vol. 104, no. 1-4, pp. 171-175, 1997.

[7] V. Kataev, G. Khaliullin, G. Michels et al., "ESR study of the electronic properties of ternary europium pnictides," Journal of Magnetism and Magnetic Materials, vol. 137, no. 1-2, pp. 157-166, 1994.

[8] J. Paglione and R. L. Greene, "High-temperature superconductivity in iron-based materials," Nature Physics, vol. 6, no. 9, pp. 645-658, 2010.

[9] F. Wang and D.-H. Lee, "The electron-pairing mechanism of iron-based superconductors," Science, vol. 332, no. 6026, pp. 200-204, 2011.

[10] H. Fukazawa, N. Takeshita, T. Yamazaki et al., "Suppression of magnetic order by pressure in $\mathrm{BaFe}_{2} \mathrm{As}_{2}$," Journal of the Physical Society of Japan, vol. 77, no. 10, Article ID 105004, 2008.

[11] H. Lee, E. Park, T. Park et al., "Pressure-induced superconducting state of antiferromagnetic $\mathrm{CaFe}_{2} \mathrm{As}_{2}$," Physical Review BCondensed Matter and Materials Physics, vol. 80, no. 2, Article ID 024519, 2009.

[12] C. Huhnt, G. Michels, W. Schlabitz, D. Johrendt, and A. Mewis, "Pressure-driven valence change in ternary Eu pnictides," Journal of Physics Condensed Matter, vol. 9, no. 45, pp. 9953-9960, 1997. 

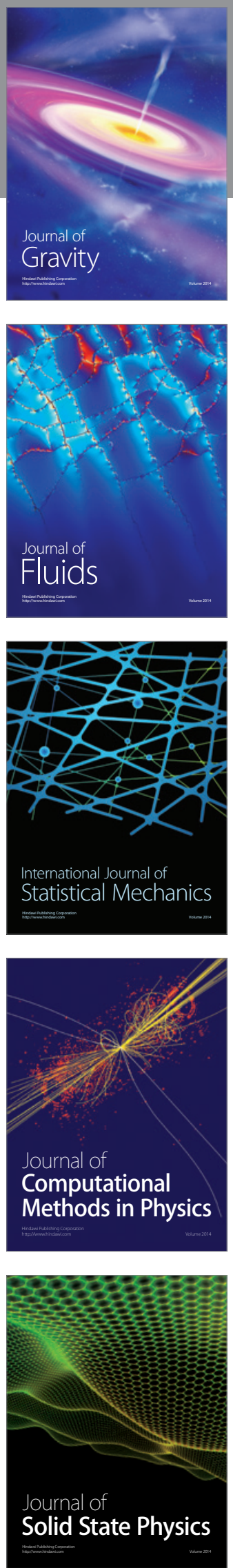

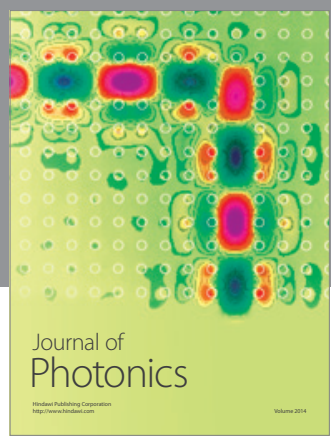

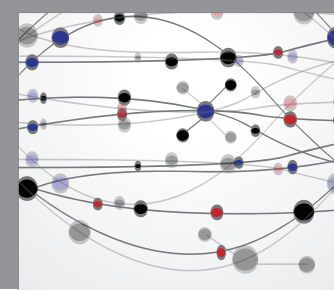

The Scientific World Journal

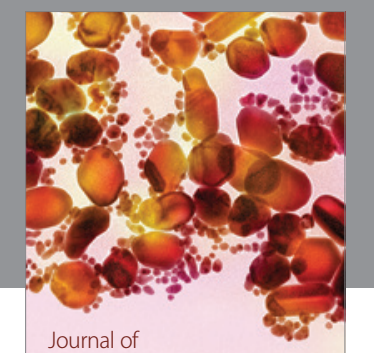

Soft Matter
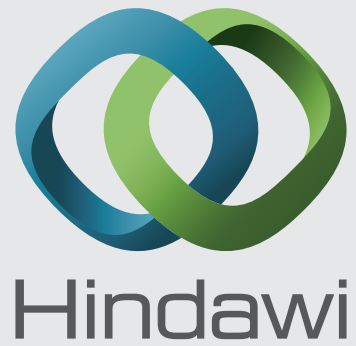

Submit your manuscripts at

http://www.hindawi.com
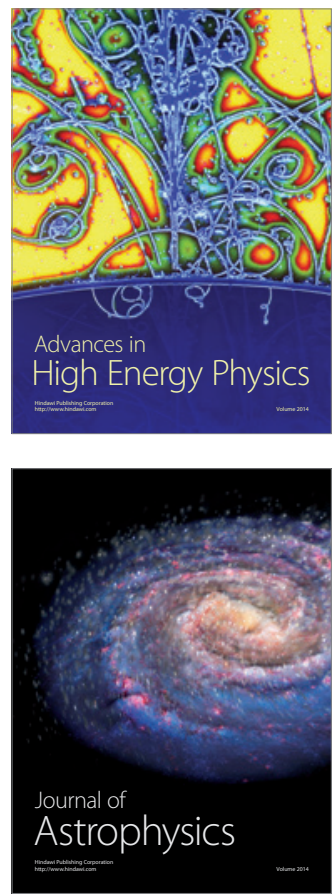
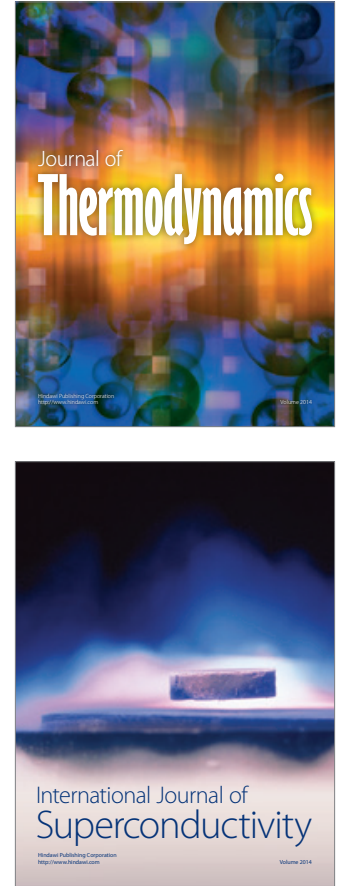
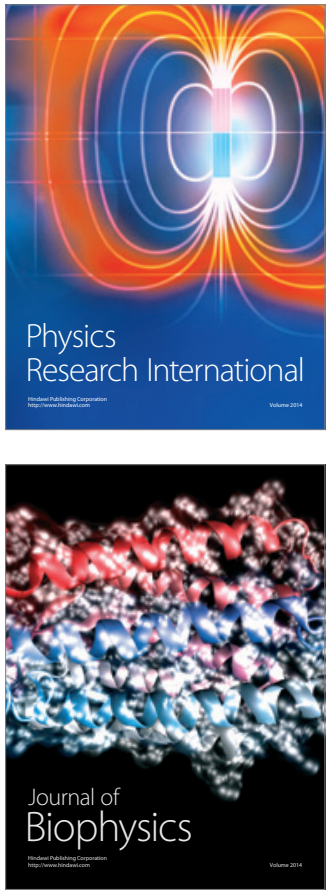
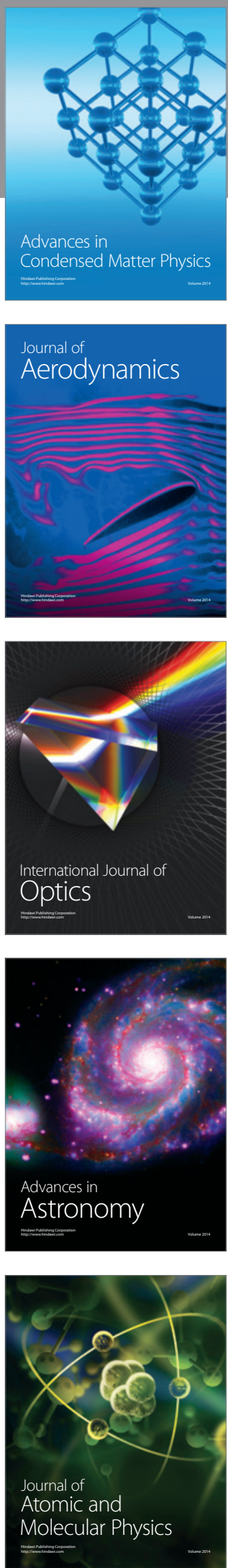